\title{
New Type Far IR and THz Schottky Barrier Detectors for Scientific and Civil Application
}

\author{
V. G. Ivanov ${ }^{1}$ and G. V. Ivanov ${ }^{2}$ \\ ${ }^{1}$ Solid State Infrared Devices, Electron, Central Research Institute, Toreza Avenue, 68, Saint-Petersburg 194223, Russia \\ ${ }^{2}$ Broadband Home Solutions, Motorola Mobility, Sedova Street 12, Saint-Petersburg 192019, Russia
}

Correspondence should be addressed to V. G. Ivanov, vivanovg@gmail.com

Received 7 April 2011; Revised 25 May 2011; Accepted 4 July 2011

Academic Editor: Satishchandra B. Ogale

Copyright (C) 2011 V. G. Ivanov and G. V. Ivanov. This is an open access article distributed under the Creative Commons Attribution License, which permits unrestricted use, distribution, and reproduction in any medium, provided the original work is properly cited.

\begin{abstract}
The results of an experimental investigation into a new type of VLWIR detector based on hot electron gas emission and architecture of the detector are presented and discussed. The detectors (further referred to as HEGED) take advantage of the thermionic emission current change effect in a semiconductor diode with a Schottky barrier (SB) as a result of the direct transfer of the absorbed radiation energy to the system of electronic gas in the quasimetallic layer of the barrier. The possibility of detecting radiation having the energy of quantums less than the height of the Schottky diode potential barrier and of obtaining a substantial improvement of a cutoff wavelength to VLWIR of the PtSi/Si detector has been demonstrated. The complementary contribution of two physical mechanisms of emanation detection- "quantum" and hot electrons gas emission-has allowed the creation of a superwideband IR detector using standard silicon technology.
\end{abstract}

\section{IR and THz Detectors for Space Astronomy}

The reception of television images of various objects in the far IR and submillimetric band of a spectrum is of great interest in solving many scientific and applied problems. Such problems can be met in astronomy, fault detection, medicine, and safety systems [1]. In recent years very close attention has been paid to the development of various $\mathrm{THz}$ systems; however, the problem of creating the most progressive staring detectors for the far IR and $\mathrm{THz}$ band of the spectrum is far from resolved [2]. Over the set of the major characteristics the most demanding requirements are imposed on staring detectors (or FPAs) for optoelectronic systems (OESs) for exoatmospheric astronomy. As a rule, if detectors of such class can be created, they can also be used for many other purposes. The published modern detectors for space OES are shown in Table 1.

The table shows the following.

(1) The size of the focal plane used within the astronomical systems is significantly larger than the photosensitive surface of any existing FPA as well as any FPA under development. The large size is extremely undesirable and is only allowable to some extent in survey system channels and narrow field of view spectrometry channels.

(2) Among the existing FPAs it would appear that the best characteristics belong to HAWAII-2 FPA, which provides output noise less than 7 electrons and the least value of NEP limited by background fluctuations. This FPA, as well as VLWIR-002, was designed using VLSI silicon technology. The production of FPAs based on VLSI silicon currently provides, and apparently will provide for the next 5 to 10 years, a competitive advantage for this type of FPA in comparison to other types over a number of major characteristics. It should be noted that at the present time only silicon technology is capable of providing FPAs on an industrial scale.

(3) A number of works have shown that the transition edge sensor (TES) will not be able to provide NEP = $10^{-20} \mathrm{~W} / \mathrm{Hz}^{1 / 2}$ (which must be achieved according to astronomical systems architects) or an appropriate dynamic range. Development of multiplexers based 


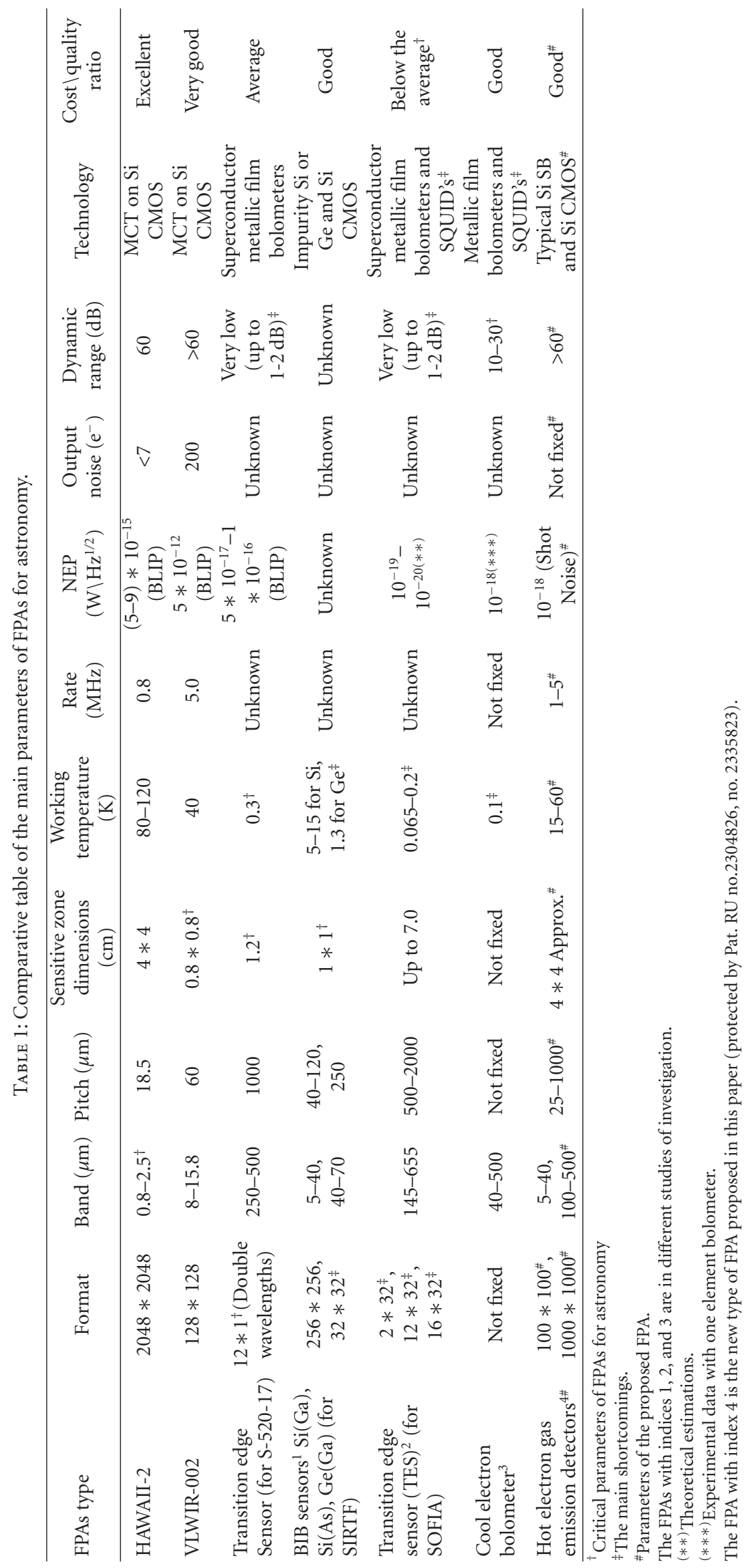


on SQUID remains 15 to 20 years behind the technology of CCD and CMOS silicon multiplexers. The obstacle of creating an on-board cooling system for this type of FPA may also be insurmountable within the timeframe of the projects being planned.

(4) Taking into account the physical principals of cool electron bolometer functioning and the results of measurements obtained for some of the detectors, detectors of this type have approached the values of the NEP given by astronomers. However, all the FPA development obstacles mentioned above for the TES remain, at least for $100 \times 100$ format FPAs.

(5) The new matrix FPA proposed in this paper, with sensitive elements based on hot electron gas emission detectors (HEGEDs) with $100 \%$ use of standard silicon technology, will resolve the problem of size such that the required values of NEP can be approached and simplify significantly the problem of FPA cooling. From the astronomical perspective, an FPA of this type will be preferable to theexisting FPA technology for on-board wide-spectrum survey cameras (SIRTF and SOFIA projects).

(6) Relatively higher operating temperatures and the use of mature silicon technology, which provides simplicity and therefore allows serial production of FPAs based on HEGED, are the undeniable advantages of the sensors in comparison to other known far IR and submillimeter radiation sensors currently used in civil equipment.

\section{New Type of IR FPA Detector}

2.1. State of the Art. Quantum infrared Schottky Barrier (IR SB) detectors, and architectures of monolithic IR FPAs based on silicon with such detectors, have been considered in many works (e.g., $[3,4])$. The sensitivity of IR SB detectors to radiation results from excitation of electrons (or holes) with absorption of radiation quantums in a quasimetallic SB layer when the energy of quantums exceeds the potential barrier [1]. The achievable cutoff wavelength of an SB photo diode's sensitivity while using such "quantum" physical mechanism of IR waves detection is completely determined by the height of the potential barrier. The increase in the cutoff wavelength $[5,6]$ has been achieved by lowering the potential barrier height. The sensitivity of such SB quantum detectors decreases quickly with increasing wavelength. Lowering the SB barrier height results in a fast increase of SB dark current which essentially worsens the sensitivity threshold of the detector and demands a significant decrease in cooling temperature, essentially lowering the dark current to reduce the shot noise and increase SNR.

Within a waveband of 12-25 microns the energy of quantums lies within the range of $0.1-0.05 \mathrm{eV}$. Creation of quantum IR SBs with these potential barrier heights is problematic.

Detectors and FPAs with VLWIR and sub-mm sensitivity can be created on the basis of thermal detectors [7]. The operation of all thermal detectors of electromagnetic emanation is based on the variation of the physical characteristics of the detector's substance at heating caused by the absorbed emanation. To provide maximum relative increase in the detector's temperature (per unit of the absorbed energy) and prevent leakage of the heat flow to the substrate, the full thermal capacity of the detector has been reduced as much as possible. In particular, such SB thermionic detectors are considered in [8].

The shortcomings of thermal detectors and FPAs with thermal detectors are the constructive and technological complexities of thermic insulation of the detector's pixels from each other and from the substrate, as well as temperature fluctuations and sensitivity to vibrations.

Recently we suggested [9] a new physical principle of operation for the IR detector (as well as a design for the detector and the FPA), where the effect of a thermionic emission current shift in a semiconductor diode with a Schottky barrier (SB) is caused by direct transfer of absorbed radiation energy to the system of electronic gas (as a hole) in the quasimetallic layer of the barrier. The opportunity to detect the radiation having energy of quantums less than the height of Schottky diode potential barrier and to obtain a substantial improvement of a cutoff wavelength to VLWIR of the PtSi/Si detector has been demonstrated [10]. Thus, the temperature of the crystalline lattice of the detector can practically stay constant, and thermic insulation of the detectors and the substrate is not required. In [9] such detectors have been referred to as hot electron gas emission detectors (HEGEDs).

2.2. New Detector's Principle of Operation. The principle of operation of detectors such as HEGED is explained by Figure 1. The cross-section of the p-type silicon detector with $\mathrm{PtSi} / \mathrm{Si} \mathrm{SB}$ is shown schematically on Figure 1(a). Figure 1(b) shows the resulting zone diagram of the detector, and Figure 1(c) shows qualitatively the dependence of the probability of the energy levels occupied by platinum silicide on the energy of electrons for three various Kelvin temperatures of the electron gas.

The electromagnetic radiation of the FIR with energy $h v$ smaller than the height of the potential barrier of the $\mathrm{SB}$ is directed through the silicon substrate (1) and the doped silicon layer (2) and is absorbed by the layer (3). The thickness of this layer (3) is comparable to the inverse absorption constant in the given silicide of metal. Within the metal silicide layer (e.g., platinum silicide) the absorption is caused mainly by the gas of the free electrons. As in the case when a SB operates as a quantum detector of radiation, the absorption of the radiation with energy $h v$ in the layer (3) results in generation of "hot" electrons (or holes), although with insufficient energy to overcome the potential barrier $\left(\Psi_{\mathrm{MS}}\right)$ of the metal-semiconductor junction. These electrons cannot directly pass from metal to the semiconductor and thus cannot create a signal current. The SB cannot be used as the quantum detector. However, the extra energy of these "hot" electrons gets transferred to all the free electrons in the metal silicide through inelastic electron-electron collisions within time $\tau_{e-e} \leq 10^{-12} \mathrm{~s}$. At the same time, at a low 
(a)
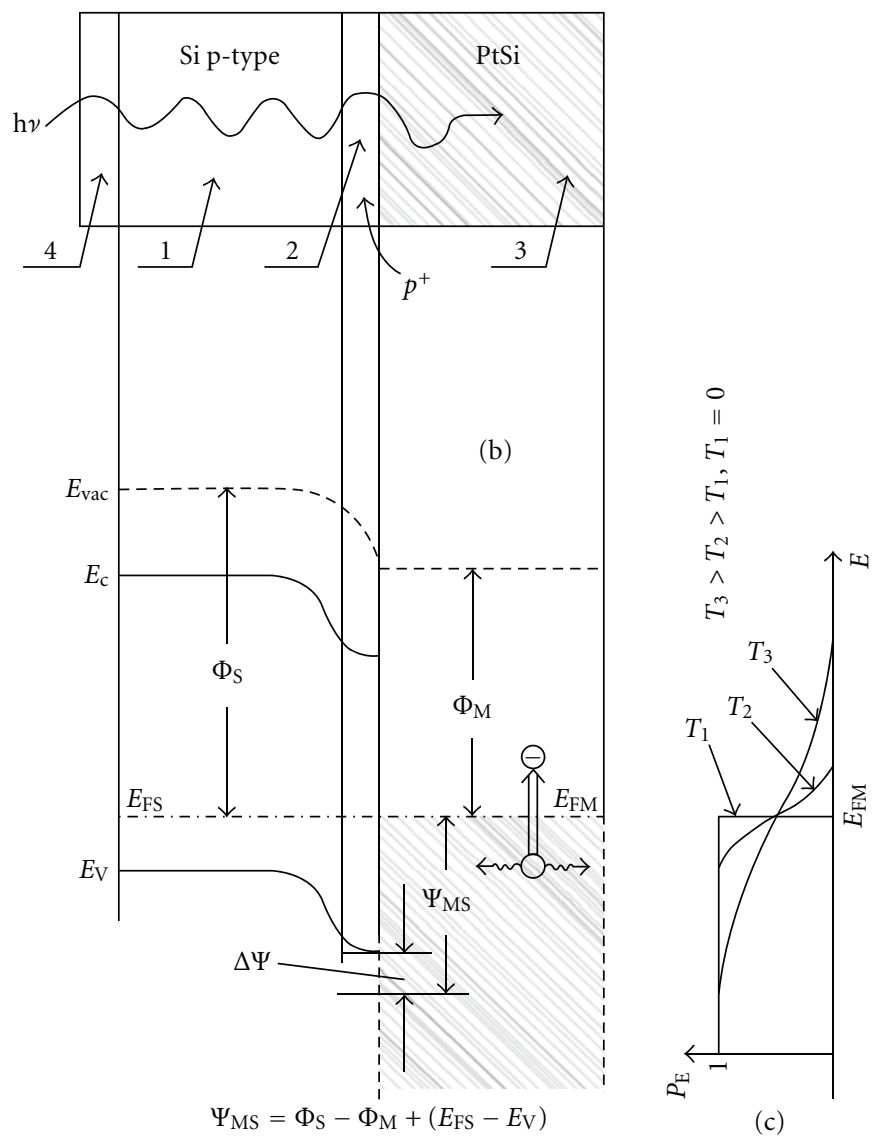

(c)

FIgure 1: Cross-sectional view and energy diagrams of the detector: (a) a cross-section of the detector on silicon of p-type with SB from platinum silicide (PtSi/Si); (1): substrate, (2): $\mathrm{p}^{+}$layer, (3): PtSi $\backslash$ Si layer, (4): antireflection coating; (b) the zone diagram of the detector ( $E_{\mathrm{vac}}$ : energy level of vacuum, $E_{c}$ : bottom of conductance band, $E_{v}$ : a top (ceiling) of valence band, $E_{\mathrm{FS}}, E_{\mathrm{FM}}$ : Fermi energy in the semiconductor and in metal, accordingly, $\Phi_{S}, \Phi_{\mathrm{M}}$ : potentials of work function from the semiconductor and metal, $\Psi_{\mathrm{MS}}$ : maximal SB barrier height, and $\Delta \Psi$ : depression of barrier height at the reverse direction voltage; (c) the dependence of probability of occupancy of energy levels of platinum silicide from electrons energy for three different temperatures of electronic gas.

temperature the electron-phonon energy relaxation time appears to be much longer and lies within $10^{-7}-10^{-10} \mathrm{~s}$. At this condition an electron gas temperature increase has been observed which results in a growth of the probability of the states where the energy levels greater than $\Psi_{\mathrm{MS}}$ are occupied by holes (in the p-type silicon) and, therefore, an occurrence of an additional current of thermionic emission from metal into the semiconductor. The difference between the currents of the thermionic emission at absorption of the radiation and without the absorption creates a signal current as in [8].

The key point of the signal current appearance is the creation of conditions where the spatial-energetic equilibrium in the system of electron gas in the layer of metal silicide occurs much faster than the transmission of superfluous energy of this gas into the crystalline lattice (and, hence, heating up of silicide layer and silicon). In a stationary case the size and space distribution of electron gas temperature $T_{e}$ can be obtained by solving the power balance equation of the absorbed radiation by a layer and the power of all losses.

The estimations [9] for detectors $\mathrm{PtSi}-/ \mathrm{p}$-Si of $1 E-5 \mathrm{~cm}^{2}$ have shown that within the band of wavelengths of 12-19 microns and at the background temperature equal to $300 \mathrm{~K}$ the background limited mode (BLIP) can already occur at a temperature of the crystalline lattice less than or equal to $100 \mathrm{~K}$ and the threshold power corresponding to this mode (at an integration time of $40 \mathrm{~ms}$ ) can achieve (1-5) $E-15 \mathrm{~W}$. Reduction of the background load essentially reduces the value of the threshold power.

2.3. Experimental Results. A set of different samples of SB detectors based on PtSi/p-Si have been manufactured. No specific thermoinsulation measures have been undertaken between the detectors and the substrate. Except for the sizes and some details of manufacturing techniques these samples are, in fact, photosensitive elements of two-dimensional CCD FPA. Its configuration and measurement techniques are presented in [10].

Typical spectral dependencies of detector responsivity $S(\lambda)$ on the thickness of the platinum silicide layer equal to $30 \mathrm{~nm}$ at two temperatures are shown in Figure 2.

At $T=80 \mathrm{~K}, S(\lambda)$ falls with a growth of $\lambda$ and looks typical for quantum detectors with PtSi/p-Si SB. The optical 


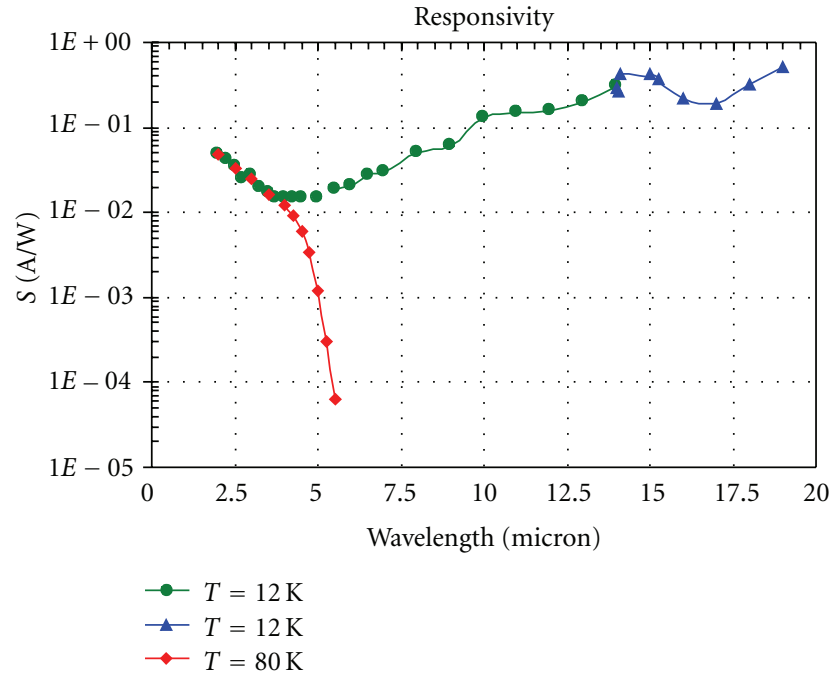

FIGURe 2: Spectral dependence of responsivity of PtSi/Si SB detectors with thickness of platinum silicide $300 \AA$ at different temperatures of crystalline lattice (reverse bias is equal to $12 \mathrm{~V}$ ).

barrier height $\Psi_{\text {opt }} \approx 0,23 \mathrm{eV}$. At excess of a wavelength of 5.5 microns the responsivity becomes less than $6 \times$ $10^{-5} \mathrm{~A} / \mathrm{W}$ for the whole examined band of wavelengths, and the "quantum" mechanism of detectors sensitivity ceases to work.

The $S(\lambda)$ dependence changes radically when the temperature decreases. Thus, at $T=12 \mathrm{~K}, S(\lambda)$ does not vary qualitatively within the waveband of 2,0-5,0 microns in comparison to the observed data at $80 \mathrm{~K}$ and reaches its minimum at approximately 3,5-4 microns. However, with growth of the wavelength the $S(\lambda)$ grows nearly exponentially and at $\lambda=19$ microns achieves the values of $0,3-0,4 \mathrm{~A} / \mathrm{W}$. Such values of $S(\lambda)$ were previously observed for detectors with $\mathrm{PtSi} / \mathrm{p}-\mathrm{Si} \mathrm{SB}$ only at the waveband of "quantum" mechanism of sensitivity at $\lambda=2,0$ microns where they correspond to quantum efficiency $\eta=0,2$ (some decreasing of $S(\lambda)$ in a range of 16-17 microns is explained by the twophonon absorption in silicon). Such behaviour of $S(\lambda)$ means that there is a contribution of another, not quantum, physical mechanism of sensitivity. Existence of a minimum in the spectral dependence $S(\lambda)$ is associated with the contribution of both physical mechanisms into the signal current, and its depth decreases with an increase of platinum silicide layer thickness. Thus, at $12 \mathrm{~K}$ the detectors investigated show the presence of sensitivity in a wide spectral band ranging from 2 up to 19 microns, which includes the atmosphere band of transparency and such interesting absorption bands as those of water steam and carbonic gas.

It is known that for the backside illumination the shortwave limit of sensitivity for such detectors will be within the range of 1,2 micron. At the same time, at $\lambda=19$ microns the recession of $S(\lambda)$ is not observed and one can assume that further growth of $\lambda$ should be accompanied by an increase of $S(\lambda)$. Since the absorption of the radiation occurs in the metallic layer, we believe that HEGED mode can ensure that detectors and FPAs are applicable for operation over a superwideband of spectrum (up to sub-mm wavelengths).

\section{Noise Equivalent Power (NEP)}

It is well known that NEP and detectivity are the commonly accepted characteristics that are used to compare various FPAs by their ability to register the lowest (threshold) power incident upon a photosensitive element. Both of these characteristics, however, are not measurable directly and their values cannot be calculated without information about the FPA architecture and the modes at which the values of the optical signal threshold power are obtained. In particular, it is important to know which of the noise components prevail for the given FPA, its noise frequencies, and the rate of the output signal. Unfortunately, the values of these characteristics are not mentioned for most cases of FPA comparison. Therefore, attempts to compare FPAs using only the NEP values often appear to be noninformative and can sometimes even be misleading. NEP can be calculated in case the SNR and the power of desired incident signal values have been measured. However, the calculation will involve additional characteristics of the FPA mentioned above. Thus, the threshold power calculated for a photosensitive element becomes the primary characteristic obtainable from the given real measurements. Besides, this particular characteristic is very important for the purpose of sensitivity comparison of different thermal imaging systems. However, it is a matter of preference on what kind of noise equivalence is chosen, the threshold power is considered to be preferable. The above consideration is valid for NETD as well.

Theoretical values of the IR threshold power for an FPA with HEGED, operating in the mode of signal integration for $40 \mathrm{~ms}$ and with an output rate of $1 \mathrm{MHz}$, are provided in [9]. Both the detector's dark current noise and the noise created by the background electrons are taken into account. These estimations show that, for an FPA with HEGED without a background at $T<60 \mathrm{~K}$, the achievable threshold power can be $10^{-18}$ to $10^{-19} \mathrm{~W}$. The presence of a background essentially increases the threshold power. In the given research no direct noise measurements are conducted and real values of thermal background are presented. At these experimental data conditions the estimation of the threshold power at the operational mode of the detectors is similar to that described in [9] and for the spectrum band of 14 to $19 \mu$ gives a value of the order of $10^{-14} \mathrm{~W}$. It is expected that within the rate of the output signal up to several $\mathrm{MHz}$ the noise created by fluctuations of the dark charge will prevail; therefore, for an output rate of $1 \mathrm{MHz}$ we should achieve $\mathrm{NEP} \approx 10^{-17} \mathrm{~W} / \mathrm{Hz}^{1 / 2}$.

\section{An Opportunity to Increase the Detector's Operating Temperature}

The previous considerations lead us to expect that with an increase in the detector's crystalline lattice temperature its responsivity for the wavelengths with energy of quantums 
less than the barrier height should also increase. It is possible to come to the same conclusion using a theoretical approach. One should take into account that within the validity limits of Richardson's law the dependence of thermionic current, generated by both the temperature of the lattice and the temperature of electronic gas, is described by identical expressions that do not have extremes at the corresponding temperatures. The expressions also allow an estimation of the values of threshold temperature of the electron gas required to obtain the detector's SNR equal to 1 at different temperatures of the lattice. These estimations [9] show that the difference between the required threshold temperature of the electron gas and the lattice temperature decreases with the rise of the lattice temperature.

However, there are two factors that limit the opportunity to increase the operating temperature of the detector. First, with the increase of temperature there is a reduction of electron-phonon relaxation time. Thus, hot electrons transmit their energy to the lattice before they create a photocurrent, and the physical mechanism upon which HEGED is based ceases. The extrapolation estimations made in [9] for metallic layer Hf show that comprehensible values of the electron-phonon relaxation time can be obtained up to a temperature of 60 to $70 \mathrm{~K}$. The definite influence on the reduction of this "cooling" time of the electron gas can be introduced also by the structure and impurity of the metal silicide layer. Therefore, optimization of the nanostructure parameters and a certain level of cleanliness of this layer should play an important role. At this stage of the research the main focus of attention is on the problem of detection of the HEGED effect. For this purpose, obviously, temperatures lower than those supported by the theory were used. There was no sensitivity observed in the far IR at a temperature $T>30 \mathrm{~K}$.

Secondly, apart from the radiation which creates the signal photocurrent, with the presence of background radiation there is also a background photocurrent. As a result, the noise, which is determined by fluctuations of this current, depends on the responsivity of the detector. At the observance of HEGED physical mechanism operating conditions, it is reasonable to derive the absolute limit of the detector's operating temperature $\left(T^{*}\right)$ from the dark current and background photocurrent equality condition. For the multielement FPA, operating in charge integration mode, it is necessary that the sum of the dark and background charge does not result in an overflow of the pixel charge capacity. In this case the optimum operating temperature can become (and it becomes in some cases) below $T^{*}$.

\section{Conclusion}

Experimental research into detectors with PtSi/p-Si SB has confirmed the earlier theoretical estimations which showed that superwideband IR detectors of the new type (hot electrons gas emission detectors (HEGED)) can be created using Schottky Barriers and that the detectors will be sensitive to radiation at the energy of quantums smaller than the potential barrier. Spectral measurements of the responsivity made within a band of $2-19 \mu$ have shown that detectors of this type have a sensitivity of at least 1,2 and up to 19 microns and can theoretically work within LWIR and also (probably) VLWIR bands and within the submillimeter band of electromagnetic radiation spectrum. The responsivity of detectors of this type grows with increasing wavelength and at a reverse voltage of $10-30 \mathrm{~V}$ at $12 \mathrm{~K}$; at the thickness of a platinum silicide layer no more than $300 \AA$ its values achieve $0.3-0.4 \mathrm{~A} / \mathrm{W}$ at a wavelength of $19 \mu$. The operating principle of the detectors allows, according to estimations, their operating temperature to be increased up to $60-70 \mathrm{~K}$ with improvement of the silicide layer creation technology. The possible ways that two-dimensional FPAs with HEGED for LWIR and VLWIR can be created are now under investigation.

\section{References}

[1] "New T-ray source could improve airport security, cancer detection," ScienceDaily, November. 27, 2007.

[2] "Second workshop on New Concept for Far-Infrared/Submillimeter Space Astronomy," Consensus view for NASA, College Park, Md, USA, March 2002.

[3] W. F. Kosonocky, H. G. Erhardt, G. Meray et al., "Advances in platinum silicide Schottky-barrier IR-CCD image sensors," Proceedings of the Society of Photo-Optical Instrumentation Engineers, vol. 225, pp. 69-71, 1980.

[4] T. S. Villani, W. F. Kosonoky, F. V. Shallcross et al., "Construction and performance of 320x244 - element IR CCD imager with PtSi Schottky-barrier detectors," Proceedings of International Society for Optics and Photonics, vol. 1107, pp. 9-21, 1989.

[5] T. L. Lin, J. S. Park, T. George, E. W. Jones, R. W. Fathauer, and J. Maserjian, "Long-wavelength $\mathrm{PtSi}$ infrared detectors fabricated by incorporating a doping spike grown by molecular beam epitaxy Appl," Applied Physics Letters, vol. 62, no. 25, pp. 3318-3320, 1993.

[6] B.-Y. Tsaur, C. K. Chen, and B. A. Nechay, "IrSi Schottkybarrier infrared detectors with wavelength response beyond 12 mkm," Electron Device Letters, vol. 11, no. 9, pp. 415-417, 1990.

[7] A. Rogalski, "Infrared detectors," Hayka, vol. 93, no. 8, pp. 81$157,2003$.

[8] J. E. Murguia, P. K. Tedrow, F. D. Shepherd, D. Leahy, and M. M. Weeks, "Performance analysis of a thermionic thermal detector at $400 \mathrm{~K}, 300 \mathrm{~K}$, and $200 \mathrm{~K}$," Proceedings of the Society of Photo-Optical Instrumentation Engineers, vol. 3698, pp. 361375, 1999.

[9] V. G. Ivanov, G. V. Ivanov, and A. A. Kamenev, "Multielement IR detectors based on Schottky barriers sensitive to radiation with quantum energy less than the height of the potential barrier," Journal of Optical Technology, vol. 75, no. 8, pp. 518523, 2008.

[10] V. G. Ivanov, G. V. Ivanov, A. A. Kamenev, V. A. Arutynov, R. M. Stepanov, and V. I. Panasenkov, "SB IR detectors with sensitivity in region, where quantums energy is less then the barrier height," Applied Physics, no. 1, pp. 87-93, 2010 (Russian). 

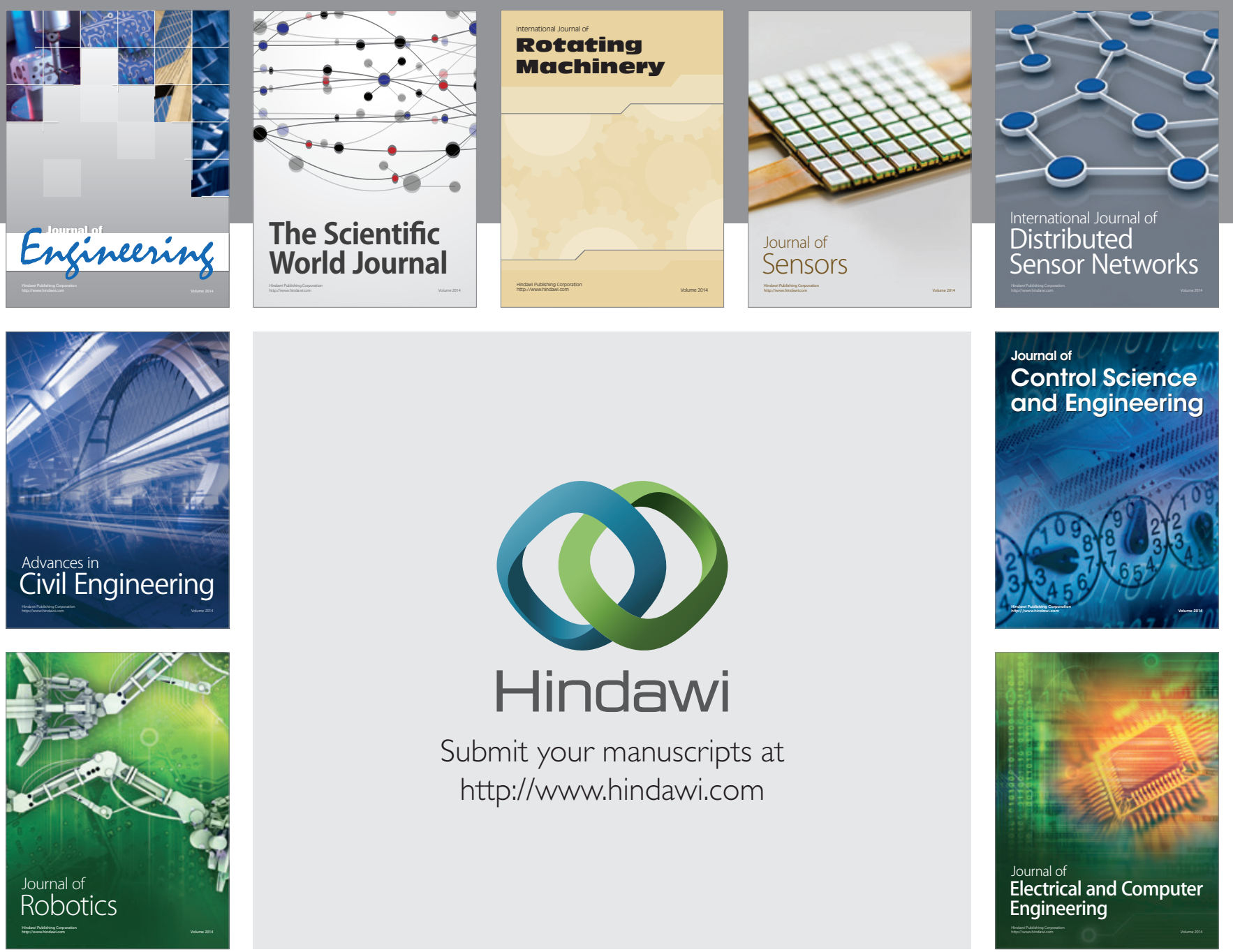

Submit your manuscripts at

http://www.hindawi.com
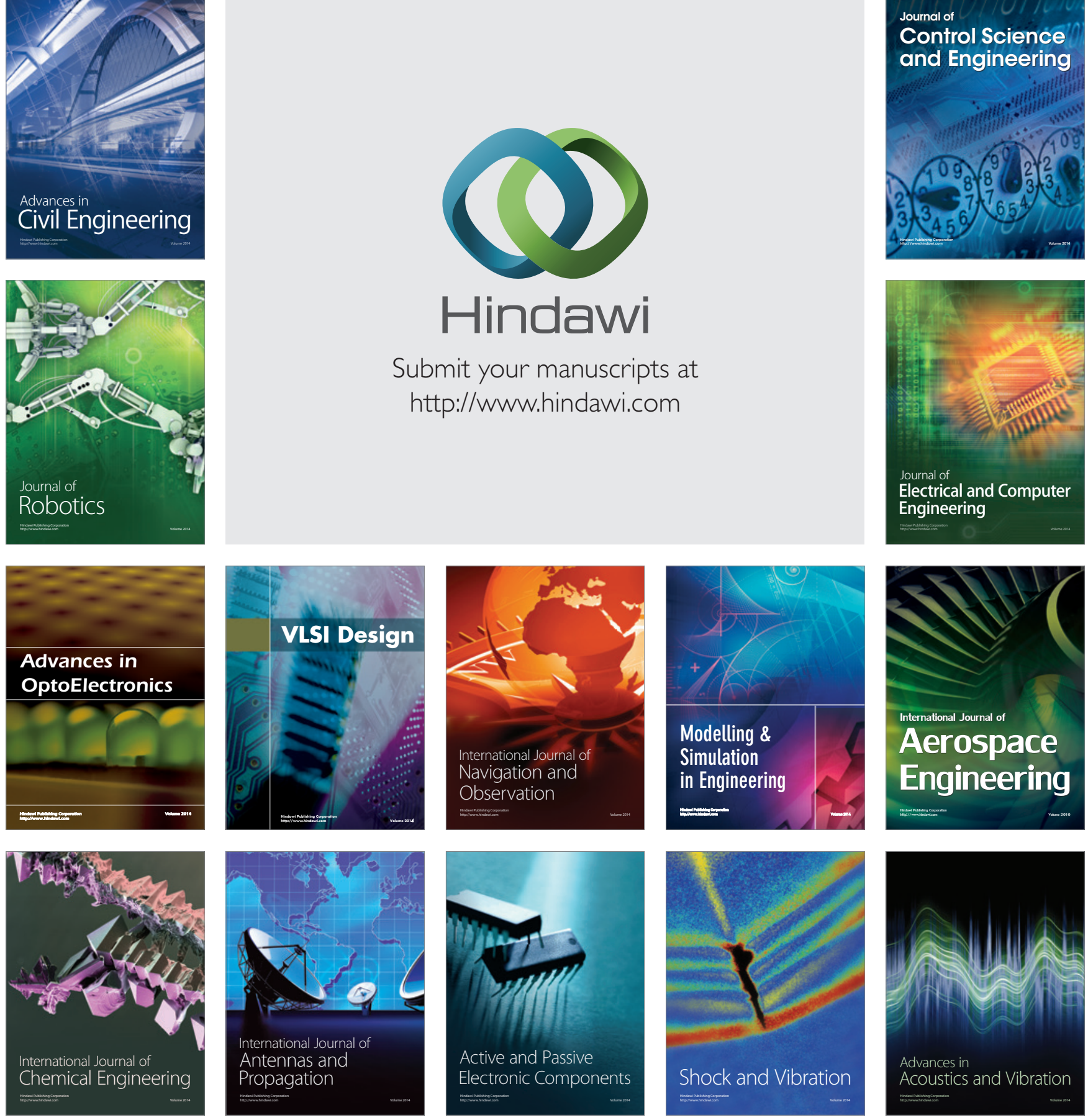